\title{
Vertebrobasilar Artery Dolicoectasia: A Case Report
}

\author{
Orkide KUTLU1 ${ }^{1}$, Hakan SELÇUK², Özlem BULUZ , Ayça KUTLU ${ }^{4}$
}

${ }^{1}$ Prof. Dr. Cemil Taşcığlu City Hospital, Department of Internal Medicine, İstanbul, Turkey

${ }^{2}$ Bakırköy Sadi Konuk Training and Research Hospital, Department of Radiology, İstanbul, Turkey

${ }^{3}$ Prof. Dr. Cemil Taşcıoğlu City Hospital, Department of Family Medicine, İstanbul, Turkey

${ }^{4}$ Istanbul University Istanbul Faculty of Medicine, Istanbul, Turkey

\begin{abstract}
Dolichoectasia is a dilative arteriopathy and often affects the intracranial vertebral and basilar arteries. Here, we present a hypertensive patient who presented with dizziness and balance disorder and was diagnosed with dolichoectasia.
\end{abstract}

Turk J Int Med 2021;3(Supplement 1):S60-S61 DOI: $\underline{10.46310 / \text { tjim.875949 }}$

Keywords: Dolicoectasia, Vertebrobasilar Artery, Headache

\section{Introduction}

Dolichoectasia is a dilative arteriopathy characterized by the lengthening, widening and bending of an artery. Intracranial vertebral and basilar arteries are the most commonly affected vessels. The incidence of vertebrobasilar artery dolichoectasis (VBD) in the population has been reported $0.06-5.8 \%$ range. Most cases are asymptomatic and diagnosed by cranial imaging performed for other reasons. Risk factors are hypertension and age over 40 years. Symptomatic patients show cerebral ischemia, bleeding, or compression of the brainstem/third ventricle/ cranial nerve roots. VBD is a rare cause of trigeminal neuralgia. One of the theories for pathogenesis of VBD; loss of elastic tissue due to early fragmentation or degeneration of internal elastic lamina; accompanying smooth muscle atrophy. Another theory is, it occurs as a complication of atherosclerosis. The extension of the artery lateral to the clivus or dorsum sella, and its bifurcation on the suprasellar cistern is considered to be elongated (dolicho). If the basilar artery diameter is over $4.5 \mathrm{~mm}$, it is considered to be enlarged (ectasic). ${ }^{1-3}$ In this case report, we aimed to present the detection of VBD in a hypertensive patient who presented with dizziness and balance disorder, since it is a rare condition.

Received:February 17,2021;Accepted:March 3,2021; Published Online:March 6, 2021 


\section{Case Report}

$67 \mathrm{y}$, female with type 2 diabetes mellitus, hypertension managed with medical treatment (metformin, clopidogrel, amlodipine, atorvastatin, ginkgo biloba). The patient had complaints of dizziness, tinnitus, and numbness in her hands and feet. Physical examination was normal. HbAlc: 6.4\%, LDL: $166 \mathrm{mg} / \mathrm{dL}$ (60-130), 25-OH vitamin D: $8.51 \mathrm{ng} / \mathrm{mL}$ (30-80), LDH: 314 U/L (0-247). Sedimentation, CRP, TSH, count blood count, vitamin B12, AST, ALT, GGT, urea, creatinine, electrolytes, and urinalysis was normal. In bilateral carotid-vertebral artery doppler ultrasonography, partially calcified echogenic plaque with a stenosis rate -not exceeding $50 \%$ - was observed in the posterior wall of the right bulbus. Local diffuse enlargement was noted at the top of the basilar artery in cranial MRI. Six months later, contrastenhanced cervical MR angiography showed normal traction, contour, flow signal patterns of the bilateral internal and external carotid arteries, and mild contour irregularities were observed in the right bifurcation region due to plaques, without significant stenosis. In cranial arterial MR angiography, local enlargement of approximately $6 \mathrm{~mm}$ in size in the anterior contour of the artery, which could not be clearly identified on the top of the basilar artery on MIP images, was noted in the raw images, and the findings were primarily thought to be aneurysm; therefore, it was recommended to be evaluated with conventional angiography. After this stage, the patient was consulted with neuroradiology in another center to clarify the need for conventional angiography. In time-of-flight (TOF) cranial MR angiography; it has been shown that the vertebrobasilar system and both internal carotid arteries (ICA) have an appearance compatible with dolichoectatic changes, it has been determined that there is no aneurysmatic condition and conventional angiography is not required. The patient will be followed clinically and angiographically.

\section{Discussion}

Dolichoectasis; describes pathological long, dilated, tortuous cerebral arteries. ${ }^{4}$ Traditionally, the diagnosis of VBD was made by catheter angiography. However, VBD diagnosis can now be made by non-invasively with $\mathrm{CT}$ and $\mathrm{MR}$ angiography imaging. MR angiography is the most sensitive imaging method; high resolution and thin section $\mathrm{T} 1$ and $\mathrm{T} 2$ weighted spin echo sequences, three-dimensional TOF MR angiography, 3D CISS sequence are the most effective sequences in the evaluation of VBD. In addition, differential diagnosis of VBD from pathologies such as aneurysms and other vascular problems, demyelinating diseases, and spaceoccupying lesions can be easily made with MR imaging.

\section{Conflict of Interests}

Authors declare that there are none.

\section{Acknowledgment}

This study has been presented in $17^{\text {th }}$ Uludag Internal Medicine National Winter Congress, $6^{\text {th }}$ Bursa Family Medicine Association National Congress, $11^{\text {th }}$ Uludag Internal Medicine Nursing Congress, 5-7 March 2021, Bursa, Turkey.

\section{References}

1. Alemdar M. Vertebrobasilar artery dolichoectesia and pain; coincidence or etiology?. Agri. 2017 Jul;29(3):1416 (in Turkish). doi: 10.5505/agri.2016.29200.

2. Yürekli VA, Gürkas E, Orhan G, $\square$ engeze $N$. Vertebrobasilar and bilateral karotid dolichoectasia: a rare entity. Turkish Journal ofCerebrovascularDiseases. 2013;19(3):112-5. doi: 10.5505/tbdhd.2013.32932.

3. Kaplan Y, Kamislı Ö, Altınayar S. Cluster-like headache associated with vertebrobasilar dolichoectasia. Turkish Journal of Cerebrovascular Diseases. 2014; 20(3): 1125. doi: 10.5505/tbdhd.2014.93064.

4. Ünlü N. Imaging findings of vertebrobasilar dolichoectasia as a cause of trigeminal neuralgia. Journal of Ankara University Faculty of Medicine. 2011;64(3);133-5 (in Turkish). doi: 10.1501/ Tipfak_000000797. 